\title{
Electrical Properties of Pristine and Electron Irradiated CNT Yarns at Small Length Scales: An Electron Microscopy Study
}

\section{Francisco Solá}

NASA Glenn Research Center, Materials and Structures Division, Cleveland, OH 44135, USA.

Carbon nanotube (CNT) yarns are currently been investigated in several NASA centers for panel applications. One critical aspect of the project is to improve the mechanical properties of this material. One potential route to achieve the mechanical improvement is the cross-linking method induced by electron beam irradiation. Ideally cross-linking between adjacent CNTs within the yarns is desirable, however cross-linking between internal walls of isolated CNTs is also possible, and both will increase the mechanical response of CNT yarns. The study of the electrical response of CNT yarns as a function of electron dose can be a complementary route to monitor possible cross-linking events, and may be important to establish multifunctionality. In this work, small segments of CNT yarns were exposed to ebeam irradiation in a transmission electron microscope (TEM) at $200 \mathrm{keV}$ and different doses. The electrical response (e.g. resistivity) as a function of e-beam irradiation was studied by the two probe method, using micromanipulators inside a scanning electron microscope. Microstructural changes were monitored by high resolution TEM. 
Microsc. Microanal. 19 (Suppl 2), 2013 